\title{
7 Prostataprobleme
}

\author{
Martin Ludwig und Axel Hegele
}

\subsection{Prostatitissyndrom}

\subsubsection{Definition}

Der Begriff „Prostatitissyndrom“ umschreibt die Symptome einer Gruppe von Patienten mit verschiedenartigen urogenitalen, perinealen und perianalen Beschwerden. Während die akute Prostatitis als schwere systemische Infektion keine diagnostischen Probleme bereitet, läßt die klinische Symptomatik bei den chronischen Prostatitisformen eine eindeutige Differenzialdiagnose zumeist nicht zu. Unter Schirmherrschaft der „National Institutes of Health“ (NIH) wurde eine Klassifizierung erarbeitet (Nickel 1998), die insbesondere die Problematik des (chronischen) Beckenschmerzes integriert und mittlerweile internationalen Standard darstellt (s. Tab. 1).

Neuere epidemiologische Daten zur Prävalenz der akuten Prostatitis deuten an, daß eine zumindest subklinische Begleitprostatitis bei 90\% aller Männer mit akutem Harnwegsinfekt auftritt. Diagnostik und Therapie der akuten Prostatitis (NIH I) bieten in der Regel kein Problem und folgen den allgemeinen Regeln der Behandlung akuter Harnwegsinfekte, so daß im Rahmen dieses Buchkapitels auf die Beschreibung dieser in der urologischen Notfallmedizin beheimateten Erkrankung verzichtet werden kann.

Andererseits ist die Bedeutung der chronischen Prostatitis/des chronischen Beckenschmerzsyndroms NIH IIIA und IIIB (CP/CPPS = chronic pelvic pain syndrome) in der urologischen Praxis belegt: Zusammenfassend wird die Prävalenz des Prostatitissyndroms in der Bevölkerung auf 5-10\% geschätzt (Schaeffer et al. 2006). Patienten mit Prostatitissymptomen in der Anamnese zeigen ein deutlich erhöhtes Risiko, weitere symptomatische Episoden und symptomatische BPH zu entwickeln.

Die asymptomatische inflammatorische Prostatitis (NIH IV) wird im Allgemeinen als Zufallsbefund im Rahmen einer Fertilitätsabklärung oder bei Prostatabiopsien zum Prostatacarcinomausschluß diagnostiziert. Eine Therapie ist nicht erforderlich. 
Tab. 1 Klassifikation des Prostatitissyndroms nach NIH

\begin{tabular}{|c|c|c|c|}
\hline Kategorie & Name & Beschreibung & $\begin{array}{l}\text { Geschätzter Anteil } \\
\text { (Schneider et al. 2003) }\end{array}$ \\
\hline I & akute bakterielle Prostatitis & akute Infektion der Prostata & nicht bekannt \\
\hline II & chronische bakterielle Prostatitis & rezidivierende Infektion der Prostata & $5-10 \%$ \\
\hline III & $\begin{array}{l}\text { chronische Prostatitis/ } \\
\text { "chronisches Schmerzsyndrom } \\
\text { des Beckens“ (CPPS = chronic } \\
\text { pelvic pain syndrome) }\end{array}$ & keine nachweisbare Infektion & \\
\hline III A & $\begin{array}{l}\text { entzündliches chronisches } \\
\text { Schmerzsyndrom des Beckens }\end{array}$ & $\begin{array}{l}\text { Leukozyten in Ejakulat, Prostatasekret } \\
\text { oder Urin nach Prostatamassage }\end{array}$ & $30-40 \%$ \\
\hline III B & $\begin{array}{l}\text { nichtentzündliches chronisches } \\
\text { Schmerzsyndrom des Beckens }\end{array}$ & $\begin{array}{l}\text { keine Leukozyten in Ejakulat, } \\
\text { Prostatasekret oder Urin nach } \\
\text { Prostatamassage }\end{array}$ & $60-70 \%$ \\
\hline IV & $\begin{array}{l}\text { asymptomatische entzündliche } \\
\text { Prostatitis }\end{array}$ & $\begin{array}{l}\text { keine subjektiven Symptome, } \\
\text { entdeckt durch Prostatabiopsie oder } \\
\text { durch Leukozyten in Prostatasekret } \\
\text { oder Ejakulat im Zuge einer } \\
\text { Diagnostik aus anderen Gründen }\end{array}$ & nicht bekannt \\
\hline
\end{tabular}

\subsubsection{Pathogenese und Ätiologie}

Bei der seltenen chronischen bakteriellen Prostatitis (5-10\% der chronischen Prostatitisformen) ist die ätiologische Bedeutung von Harnwegsinfektserregern, in erster Linie E. coli, unumstritten. Die ätiologische Relevanz von Chlamydia (C.) trachomatis und Mykoplasmenspezies wird auch heute noch kontrovers diskutiert. Anaerobier werden nur bei 1\% der Patienten gefunden und gelten ebenso wie Neisseria gonorrhoeae, Trichomonas vaginalis, Viren und Pilzspezies nur in Einzelfällen als klinisch relevant. Mycobacterium tuberculosis kann im Rahmen einer Urogenitaltuberkulose eine Prostatitis verursachen. An pathogenetischen Faktoren gilt bei der CBP (synonym: NIH II) der Reflux infizierten Urins in die Prostatagänge als gesichert.

Beim chronischen Beckenschmerzsyndrom (NIH IIIA und IIIB) werden vielschichtige ätiologische Aspekte kontrovers diskutiert, die in Tabelle 2 zusammengefasst sind.

\subsubsection{Symptomatik}

Der Beschwerdedruck von Patienten mit chronischer Prostatitis ist hoch und gemessen mit validierten Beschwerdeskalen vergleichbar mit Patienten, die an instabiler Angina pectoris, aktivem M. Crohn oder Z.n. Herzinfarkt leiden. Die Symptomatologie der chronischen Prostatitis umfaßt typische Schmerzsyndrome des Beckens (z.B. retropubische oder perineale Beschwerden), Entzündungssymptome (z.B. Brennen bei Miktion oder Ejakulation), irritative und/oder obstruktive Miktionsstörungen (Pollakisurie, Harndrang, abgeschwächten Harnstrahl), sowie Störungen der Sexualfunktionen (erektile Dysfunktion, Ejaculatio präcox). 
Tab. 2 Pathogenetische Faktoren des chronischen Beckenschmerzsyndroms (NIH III A und B)

\begin{tabular}{|c|c|}
\hline Pathogenese & Bemerkung \\
\hline (post-)infektiös & $\begin{array}{l}\text { Nachweis bakteriellen Genoms im Prostatagewebe } \\
\text { durch Nanobakterien verursachte Entzündung oder Prostataverkalkungen }\end{array}$ \\
\hline analog zur interstitiellen Cystitis & Epithelschaden mit Störung der Glycosaminoglycanschicht der Harnblase \\
\hline physikalisch & Reflux von Urin und stickstoffhaltigen Urinprodukten in die Prostatagänge \\
\hline (auto-)immunologisch & $\begin{array}{l}\text { Auto-Antikörper (PSA?) } \\
\text { Zytokinproduktion } \\
\text { Mastzelldegranulation } \\
\text { T-Zell vermittelte Autoimmunität }\end{array}$ \\
\hline psychogen & $\begin{array}{l}\text { Stress-Prostatitis } \\
\text { „Angst-Spannungs-Zyklus“ }\end{array}$ \\
\hline genetisch & X-chromosomale Deletionen \\
\hline $\begin{array}{l}\text { funktionelle/morphologische } \\
\text { subvesikale Obstruktion }\end{array}$ & $\begin{array}{l}\text { verstärkter intraprostatischer Druck } \\
\text { Innervationsstörung der glatten/quergestreiften Muskulatur } \\
\text { Beckenbodenmyalgie } \\
\text { Blasenhalshypertrophie }\end{array}$ \\
\hline neurogen & Senkung der Schmerzschwelle \\
\hline Veränderungen im Analbereich & $\begin{array}{l}\text { „Anogenitalsyndrom“ } \\
\text { Hämorrhoiden, Fissuren }\end{array}$ \\
\hline hoher Samenwegsverschluss & Utriculuszyste (selten!) \\
\hline
\end{tabular}

Komplizierte Verflechtungen mit subvesikaler Obstruktion, sexueller Dysfunktion und chronischen Schmerzzuständen erfordern den Einsatz standardisierter Fragebögen, um spezifische „Prostatitis-Symptome“ qualitativ und quantitativ evaluieren zu können. Im deutschen Sprachraum steht dabei die deutsche Version des NIH-CPSI (National Institutes of Health - Chronic Prostatitis Symptom Index) zur Verfügung (Hochreiter et al. 2001). Der IPSS (International Prostate Symptom Score) evaluiert darüber hinaus eine in $50 \%$ vorhandene begleitende funktionelle oder anatomische Blasenentleerungsstörung.

\subsubsection{Diagnostik}

Es hat sich bewährt, die diagnostischen Optionen beim CPPS gemäß ihrer Notwendigkeit und Effizienz einzuteilen (Wagenlehner et al. 2009a). In der klinischen Routine werden unterschieden:

- empfohlene Diagnostik (Basisdiagnostik)

- optionale Untersuchungen

- nicht routinemäßig durchzuführende Untersuchungen 


\section{Basisdiagnostik}

Die Notwendigkeit einer standardisierten quantitativen und qualitativen Symptomevaluation mittels skalierter Fragebögen zu Diagnosefindung und Therapieverlauf wurde bereits betont. Der in validierter deutscher Übersetzung (Hochreiter et al. 2001) vorliegende NIH-CPSI hat drei Teilbereiche (Schmerz, Miktion, Lebensqualität). Der Unterbereich „Schmerz“ diskriminiert erfahrungsgemäß am besten zwischen Männern mit und ohne CP/CPPS. Ab einem Schmerz-Score von > 10 geht man im Allgemeinen von einer manifesten Prostatitis-Symptomatik aus (Schneider et al. 2005).

Das zweite zentrale Standbein der Basisdiagnostik stellt die Lokalisationsdiagnostik von Entzündung und Infektion dar. Klassischerweise wird seit 4 Jahrzehnten die sogenannte „4-Cläser-Probe“ propagiert, bei der gleichzeitig das Leukozytenaufkommen zusammen mit einer semiquantitativen Erregersuche in Erst-, Mittelstrahl-Exprimaturin sowie im exprimierten Prostatasekret durchgeführt wird. Befragungen US-amerikanischer Urologen haben allerdings gezeigt, dass aufgrund der komplizierten und zeitaufwendigen Prozedur die 4-Cläser-Probe im klinischen Alltag keine Rolle spielt. Neuere Daten belegen zudem, dass in der klinischen Routine die vergleichende Untersuchung von Urin vor und nach Prostatamassage für die Differenzialdiagnostik der einzelnen Prostatitisformen zuverlässige Ergebnisse liefert. Entscheidend ist hier eine 10-fach höhere Konzentration von Leukozyten im Exprimaturin, um eine entzündliche Prostatitis (NIH II, IIIA) zu diagnostizieren. Eine chronische bakterielle Prostatitis (NIH II) definiert sich durch eine 10-fach höhere Erregerkonzentration typischer Harnwegsinfekterreger im Exprimaturin (Ludwig et al. 2000).

\section{Optionale Untersuchungen}

Bereits seit langem ist unbestritten, dass infolge von Veränderungen in der Urethra mit Verwirbelung des laminaren Urinstroms „prostatitische Beschwerden“ auftreten können. Da sich bei 30-40\% der Patienten mit „prostatitischen Beschwerden “ urodynamisch wirksame Veränderungen zeigen, ist die Abklärung der Blasenentleerung zu empfehlen. Funktionelle Veränderungen dominieren mit 33\% gegenüber echten Obstruktionen, die nur 2\% betragen (Mayo et al. 1998). Bei pathologischen Befunden im IPSS sollten daher als Stufenschema weitere diagnostische Schritte folgen (retrogrades Urethrogramm, Urethrocystoskopie, Cystomanometrie).

Die NIH-Klassifikation fordert die Untersuchung des Ejakulats zur Differenzialdiagnose der einzelnen Prostatitisformen. Allerdings ist nur bei der chronischen bakteriellen Prostatitis in nahezu allen Fällen eine signifikante Bakteriospermie mit dem in der Standard-Lokalisationsdiagnostik („4-Gläser-Probe“) nachgewiesenen Erreger zu finden. Da 50\% aller asymptomatischen Männer eine Bakteriospermie als Zeichen einer Besiedlung der vorderen Harnröhre aufweisen, ist die alleinige mikrobiologische Ejakulatanalyse irreführend. Zudem ist die Ejakulatanalyse nicht für eine Lokalisationsdiagnostik der urogenitalen Entzündung oder Infektion geeignet. In wissenschaftlicher Hinsicht interessant, ist die Ejakulatanalyse in der klinischen Praxis häufig irreführend, sodass sie keinen Eingang in die Routinediagnostik gefunden hat.

Der transrektale Ultraschall (TRUS) kann im Einzelfall hilfreiche Befunde wie Prostatasteine oder Zysten detektieren, die Informationen über Pathogenese und Prognose der Erkrankung liefern können. Eine Diagnosestellung allein anhand sonographischer Kriterien ist allerdings nicht möglich. 


\section{Nicht routinemäßig durchzuführende Untersuchungen}

Weitere Untersuchungen wie Kaliumchlorid-Belastungstest, Nachweis von C. trachomatis oder Mykoplasmen, molekularbiolologische Erregergenom-Nachweise oder bildgebende Diagnostik wie MRT bleiben speziellen klinischen oder wissenschaftlichen Fragestellungen in spezialisierten Zentren vorbehalten.

\subsubsection{Therapie}

\section{Chronische bakterielle Prostatitis (NIH II)}

Hier steht an erster Stelle der Therapie die Antibiose, primär mit einem modernen Fluorochinolon für 2-4 Wochen. Mit dieser Therapie sind bei gramnegativen Infektionen mikrobiologische Heilungsraten von über $70 \% \mathrm{zu}$ erwarten. Aufgrund einer längeren Therapiedauer (3 Monate) und der niedrigeren Erfolgsrate gilt Cotrimoxazol als Mittel der zweiten Wahl. Inwieweit eine Normalisierung der Symptome durch eine Eradikation des Erregers erzielt werden kann, ist bisher nicht ausreichend evaluiert. Bei Versagen der Primärtherapie schließt sich eine niedrig dosierte Langzeitantibiose mit Trimethoprim oder Nitrofurantoin zur Therapie rezidivierender Harnwegsinfekte an; auch diese Therapie ist nur unzureichend evaluiert (Übersicht in Wagenlehner et al. 2009a).

Obwohl die Existenz einer mykoplasmen- oder chlamydienassoziierten Prostatitis nie definitiv bewiesen werden konnte, ist die probatorische Gabe eines potenziell wirksamen Antibiotikums (z.B. Doxycyclin 2 x $100 \mathrm{mg}$ tägl. über 2 Wochen) bei entsprechendem Erregernachweis vertretbar. Der Effekt einer derartigen Therapie ist allerdings völlig unklar.

\section{Chronisches Beckenschmerzsyndrom (NIH IIIA und B)}

Die häufigsten Therapiestrategien umfassen die antimikrobielle Therapie, Alphablocker und anti-inflammatorische Substanzen.

Antibiotika stellen die häufigste initiale Therapiestrategie bei Patienten mit CP/CPPS dar, obwohl eine bakterielle Infektion nur bei 5-10\% aller Patienten zu beweisen ist (Taylor et al. 2008). Da bei diesem Krankheitsbild auch die Möglichkeit einer mit konventionellen Methoden nicht nachweisbaren Infektion diskutiert wird, empfahl eine europäische Konsensuskonferenz den probatorischen Einsatz von Antibiotika beim entzündlichen (NIH IIIA), nicht jedoch beim nichtentzündlichen (NIH IIIB) Beckenschmerzsyndrom. Diese Empfehlung beruht jedoch nicht auf „evidenzbasierten“ Daten, da bis heute doppelblinde, randomisierte Studien auf den fehlenden symptomatischen Benefit von Männern hinweisen, die mit Fluorochinolonen therapiert wurden, sodass der Nutzen einer probatorischen antibiotischen Therapie bei diesen Patienten in Zweifel zu ziehen ist (Alexander et al. 2004).

Auch die Daten zur Therapie mit Alphablockern (Terazosin, Alfuzosin, Tamsulosin) bieten kein einheitliches Bild. Am ehesten scheinen sie bei den Patienten geeignet zu sein, die noch keine Vortherapie mit Alphablockern erhalten haben, moderate bis starke Beschwerden aufweisen und gewillt sind, die Therapie über mehr als 6 Wochen konsequent fortzuführen. Bei multipel vorbehandelten Patienten mit einer lan- 
gen Beschwerdenanamnese sollen Alphablocker jedoch keine signifikante Erleichterung erbringen (Übersicht in Wagenlehner et al. 2009a).

Bestimmte Phytotherapeutika wie Roggenpollenextrakt weisen anti-inflammatorische Mechanismen auf und haben sich, soweit untersucht, in der Therapie als signifikant wirksam erwiesen (Wagenlehner et al. 20ogb). Der Einsatz nichtsteroidaler Antiphlogistika ist zwar weit verbreitet, entbehrt jedoch weitgehend einer ,evidenzbasierten“ Datengrundlage.

Aufgrund der limitierten Therapieerfolge der genannten Therapiestrategien wurde eine Vielzahl weiterer Therapieoptionen medikamentöser, physikalischer, schmerzmodulierender, verhaltenstherapeutischer, operativer, minimalinvasiver und multimodaler Optionen entwickelt, die eine unterschiedliche Evidenzqualität aufweisen. Zumeist bleibt dabei völlig unklar, welche Therapiestrategie bei einer individuellen Befundkonstellation vorzuziehen ist. Tabelle 3 gibt einen Überblick über die wichtigsten, bisher erst ansatzweise in placebokontrollierten Studien getesteten Optionen.

Als Ursache dieser enttäuschenden Ergebnisse wird aktuell diskutiert, dass die chronische Prostatitis NIH einen Endzustand darstellt, dem ganz unterschiedliche Pathomechanismen und zusätzliche auslösende Faktoren zugrunde liegen. Eine Therapie, die unselektioniert versucht, diesen Endpunkt zu behandeln, ohne den unterschiedlichen auslösenden Faktoren Rechnung zu tragen, ist daher zum Scheitern verurteilt. US-amerikanische Autoren haben anhand der aktuellen Literatur 6 klinische Domänen formuliert, die eine klinische Phänotypisierung und sinnvolle Klassifizierung der Patienten mit CP/CPPS ermöglichen mit dem Ziel, dem individuellen Patienten eine seiner Ätiopathogenese angepasste Therapieoption anbieten zu können. Die differenzierbaren Pathomechanismen $(\mathrm{U}=$ urinogen; $\mathrm{P}=$ psychosozial; $\mathrm{O}$ = organ-spezifisch; $\mathrm{I}$ = infektiös; $\mathrm{N}$ = neurologisch/systemisch; $\mathrm{T}$ = tenderness of muscles [Beckenbodenverspannungen]) wurden als sogenannte UPOINT-Hypothese formuliert und bedingen den Endzustand CP/CPPS (Shoskes et al. 2009).

\section{Tab. 3 „Low evidence“ Therapieoptionen beim chronischen Beckenschmerzsyndrom NIH III}

$\begin{array}{llll}\text { Medikamentös } & \text { Operativ } & \text { „Physikalisch“ } & \text { Verhaltensänderungen } \\ \text { Muskelrelaxantien } & \text { Sacrale } & \text { Biofeedback, Beckenbodentraining } & \text { Stressreduktion } \\ \text { Antiphlogistika } & \text { Nervenstimulation } & \text { Akupunktur } & \text { häufige Ejakulation } \\ \text { Antibiotika + } & \text { Neodym-YAG Laser } & \text { myofasziale manuelle Triggerpunkte } & \text { Wärmeexposition } \\ \text { Alphablocker } & \text { Perisphinktere } & \text { repetitive Prostatamassage } & \text { Diät } \\ \text { Anti-Nanobakteriell } & \begin{array}{l}\text { Injektion von } \\ \text { Botulinum Toxin }\end{array} & \text { Beckenbodenrelaxation } & \\ & & \text { perkutane periphere Nervenstimulation } & \\ & & \text { urethro-anale Elektrostimulation } & \\ & & \text { elektromagnetische Therapie } & \\ & & \text { Osteopathie } & \end{array}$




\subsection{Benignes Prostatasyndrom}

\subsubsection{Definition}

Für die Beschreibung obstruktiver Miktionsstörungen des unteren Harntraktes wird eine Vielzahl von Synonymen verwendet. Am gebräuchlichsten ist die Bezeichnung der benignen Prostatahyperplasie (BPH), welche allerdings eine histologische Diagnose darstellt und deshalb vor Erhalt eines histo-pathologischen Befundes nicht eingesetzt werden sollte. Stattdessen werden die klinischen Symptome einer obstruktiven Miktionsstörung mit dem Begriff benignes Prostatasyndrom (BPS) beschrieben. Die Symptomatik des BPS kann obstruktiven, irritativen sowie gemischt obstruktiv/ irritativen Charakters sein. Die Symptome werden allgemein und unspezifisch als Symptome des unteren Harntraktes („lower urinary tract symptoms“ LUTS) zusammengefasst. LUTS bezeichnet Störungen im Zusammenspiel der Harnblase und Prostata bei der Blasenfüllung und Blasenentleerung. Eine LUTS ist meist, muss aber nicht immer durch eine benigne Prostataobstruktion (BPO) bedingt sein (Oelke et al. 2010; Fitzpatrick 2006).

\subsubsection{Anatomie}

Die Prostata ist nach McNeal aus verschiedenen Zonen aufgebaut und von einer Kapsel umschlossen. Die BPH entsteht in der Transitionalzone und in der periurethralen Zone. Durch die Nähe zur Harnröhre und dem Vorhandensein einer Kapsel kann der entstehende Druck zu einer Einengung und konsekutiv zu Problemen beim Wasserlassen führen.

\subsubsection{Epidemiologie und Ätiologie}

Die BPH ist beim alternden Mann die häufigste Ursache einer Miktionsproblematik. Die Prävalenz steigt mit dem Alter an. Im Rahmen von Autopsiestudien konnte nachgewiesen werden, dass ca. 50\% der Männer ab dem 6o. Lebensjahr und 70\% ab dem 70. Lebensjahr an einer BPH leiden. Bei Männern unter 30 Jahren zeigt sich diese hingegen nicht. Man geht davon aus, dass ab dem 50. Lebensjahr ca. 30\% der Männer an einem behandlungsbedürftigen BPS leiden (Boyle 1994). Die Anzahl der betroffenen, therapiebedürftigen Männer steigert sich mit zunehmendem Alter. Neben dem Alter stellen das Prostatavolumen, die Restharnmenge und die Symptome Risikofaktoren für ein Fortschreiten der Erkrankung dar, sodass man dieses Krankheitsbild durchaus als Volkskrankheit bezeichnen kann. Bei sich änderndem Gesundheitsbewusstsein und einer Zunahme der älteren Bevölkerung stellt die adäquate Diagnostik und Therapie des BPS bei schwindenden Ressourcen eine Herausforderung sowohl für den Arzt als auch den Patienten dar.

Histologisch lässt sich bei der BPH eine vermehrte Anzahl der epithelialen und stromalen Zellen nachweisen. Die exakte Ätiopathogenese der BPH ist unklar. Hormonelle Faktoren und deren gestörtes Zusammenspiel scheinen jedoch eine entscheidende Rolle zu spielen. Die Vergrößerung der Prostata im Alter entsteht nur bei Vorhandensein von testikulärem Androgen. In der Prostata entsteht durch das Enzym 5- $\alpha$-Reduktase (Typ 2) aus Testosteron Dihydrotestosteron (DHT), welches eine 
Tab. 4 Beschwerden des benignen Prostatasyndroms

$\begin{array}{ll}\text { obstruktive Symptome } & \text { irritative Symptome } \\ \text { Miktionsbeginn verzögert } & \text { häufige Blasenentleerung (Pollakisurie) } \\ \text { Harnstrahl abgeschwächt } & \text { nächtliche Miktion (Nykturie) } \\ \text { Miktionszeit verlängert } & \text { Drangsymptomatik } \\ \text { Restharngefühl } & \\ \text { Harnträufeln } & \end{array}$

höhere Affinität zum Androgenrezeptor aufweist. Dieser DHT/AndrogenrezeptorKomplex steuert die Transkription z.B. von Wachstumsfaktoren als Voraussetzung für eine Zellvermehrung (Oelke et al. 2005). Zusätzlich findet sich eine erhöhte BPHRate bei Söhnen oder Brüdern von betroffenen Männern, sodass auch eine familiäre Disposition bei der Entwicklung einer BPH diskutiert wird (Sanda et al. 1994).

\subsubsection{Symptomatik}

Die Vergrößerung der Prostata, mit dem Alter zunehmend, kann verschiedenartigste Symptome und Komplikationen hervorrufen, welche nur in geringem Maße mit dem Prostatavolumen korrelieren. Die Symptome können in obstruktive und irritative Beschwerden unterteilt werden (s. Tab. 4). Obstruktive Miktionsbeschwerden sind durch eine Störung der Blasenentleerung gekennzeichnet, bei irritativen Miktionsbeschwerden stehen Störungen bei der Blasenfüllung/Speicherphase im Vordergrund. Die Miktionsbeschwerden führen je nach Ausprägung und individuellem Empfinden zu einem erheblichen Leidensdruck mit konsekutiver Einschränkung der Lebensqualität. Dies kann sich durch eine Vielzahl von Komplikationen verstärken, insbesondere:

- rezidivierende Harnwegsinfekte bei Restharnbildung

- Blasensteinbildung

- Harnverhalt mit Überlaufsymptomatik

- Dekompensation des Musculus detrusor vesicae

- Hydronephrose

- postrenale Niereninsuffizienz

Schwerwiegende Komplikationen einer bestehenden Obstruktion stellen zum Beispiel die Dekompensation der Harnblasenmuskulatur mit konsekutiver Niereninsuffizienz bis hin zum terminalen Stadium und der akute Harnverhalt entweder spontan oder getriggert (z.B. durch eine Narkose, Medikamente) dar.

\subsubsection{Basisdiagnostik}

\section{Anamnese}

Erfassung der aktuellen Miktionsbeschwerden und Erfragen der typischen BPS-Symptome (Miktionsfrequenz inkl. Nykturie, rezidivierende Infekte, Harnverhalt etc.). Unerlässlich ist die Eruierung von vorangegangenen Operationen (z.B. nervaler Schaden nach Rektumchirurgie), Begleiterkrankungen (z.B. Diabetes mellitus, M. Par- 
kinson) und aktuelle Medikamenteneinnahme zur Eruierung einer extraprostatischen Ursache der Miktionsstörung.

\section{Körperliche Untersuchung}

Nach einer grob orientierenden Untersuchung des Abdomens sollte im Rahmen der Beurteilung des äußeren Genitales eine Phimose, Meatusstenose und Fehlbildungen als Ursache ausgeschlossen werden. Durch die digito-rektale Untersuchung kann die Größe und Beschaffenheit der Prostata beurteilt werden, zusätzlich dient diese der Detektion Prostatakarzinom-suspekter Areale (z.B. harter tastbarer Prostataknoten).

\section{Urinuntersuchung}

Aufgrund der Tatsache, dass LUTS auch durch Harnblasentumoren und Harnwegsinfekte bedingt sein kann, sollte eine Urinuntersuchung (z.B. Urin-Stix-Analyse) zum Ausschluss eines Infektes und einer Mikrohämaturie durchgeführt werden

\section{Prostataspezifisches Antigen (PSA)}

Da auch ein Prostatakarzinom zu LUTS führen kann und vor der Therapie des BPS ein Prostatakarzinom ausgeschlossen werden sollte ist neben der digito-rektalen Untersuchung die Bestimmung des PSA-Wertes ein wichtiger Bestandteil der Basisdiagnostik.

\section{Internationaler Prostata-Symptomen-Score (IPSS)}

Durch den international validierten, anerkannten und verwendeten IPSS-Fragebogen ist es möglich die Ausprägung der LUTS zu erfassen und in Schweregrad-Kategorien $\mathrm{zu}$ unterteilen. Anhand von 7 Fragen werden die typischen Symptome erfragt und mittels Punktescore (je o-5 Punkte, maximaler Punktwert 35) bewertet:

- 0-7 Punkte: milde Symptomatik

- 8-19 Punkte: mittelschwere Symptomatik

- 20-35 Punkte: schwere Symptomatik

Der Bogen kann mit einer Frage zur Lebensqualität ergänzt werden (s. Tab. 5). Neben dem IPSS müssen natürlich sowohl die individuelle Einschränkung der Lebensqualität als auch die klinische Symptomatik in die Entscheidungsfindung über eine Therapienotwendigkeit miteinbezogen werden. Zusätzlich kann mittels IPSS der Erfolg einer Therapie (sowohl medikamentös als auch operativ) im Verlauf kontrolliert werden.

\subsubsection{Spezielle Diagnostik}

Sollte nach Durchführung der Basisdiagnostik keine suffiziente Stellung einer Diagnose möglich sein bzw. sich relevante Änderungen der Symptomatik ergeben ist eine weiterführende Diagnostik vor Einleitung jeglicher Therapie unerlässlich. 
Tab. 5 International Prostate Symptom Score: international standardisierter Frageboden zur Quantifizierung der Beschwerden

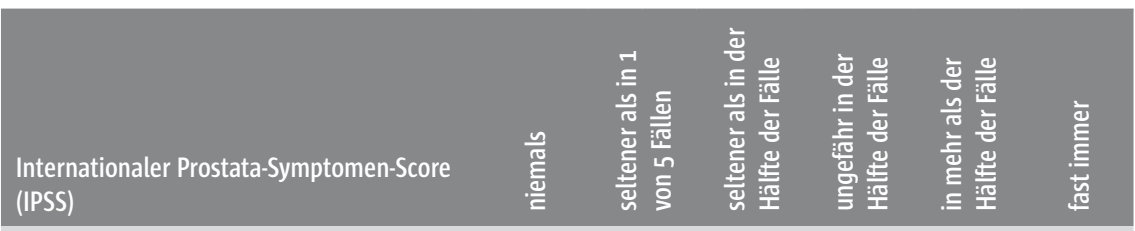

Diese Fragen beziehen sich auf Ihre Symptome in den letzten 4 Wochen

1. Wie oft hatten Sie das Gefühl, dass Ihre Blase nach dem Wasserlassen nicht ganz entleert war?

2. Wie oft mussten Sie in weniger als 2 Stunden ein zweites Mal Wasser lassen?

3. Wie oft mussten Sie mehrmals aufhören und wieder neu beginnen beim Wasserlassen?

4. Wie oft hatten Sie Schwierigkeiten, das Wasserlassen hinauszuzögern?

5. Wie oft hatten Sie einen schwachen Strahl beim Wasserlassen?

6. Wie oft mussten Sie pressen oder sich anstrengen, um mit dem Wasserlassen zu beginnen?

\begin{tabular}{|c|c|c|c|c|c|c|}
\hline 0 & & 1 & 2 & 3 & 4 & 5 \\
\hline 0 & & 1 & 2 & 3 & 4 & 5 \\
\hline 0 & & 1 & 2 & 3 & 4 & 5 \\
\hline 0 & & 1 & 2 & 3 & 4 & 5 \\
\hline 0 & & 1 & 2 & 3 & 4 & 5 \\
\hline 0 & & 1 & 2 & 3 & 4 & 5 \\
\hline niemals & & mal & zweimal & dreimal & viermal & $\begin{array}{c}\text { fünfmal } \\
\text { oder } \\
\text { mehr }\end{array}$ \\
\hline 0 & & 1 & 2 & 3 & 4 & 5 \\
\hline 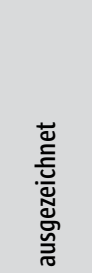 & 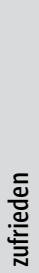 & 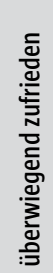 & 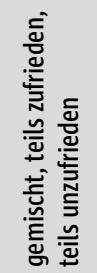 & 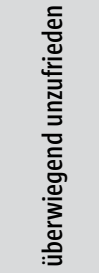 & 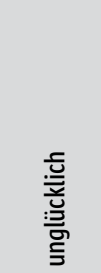 & 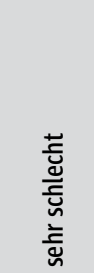 \\
\hline 0 & 1 & 2 & 3 & 4 & 5 & 6 \\
\hline
\end{tabular}

\section{Gesamt IPSS Score}

Beeinträchtigung der Lebensqualität durch Harntraktsymptome

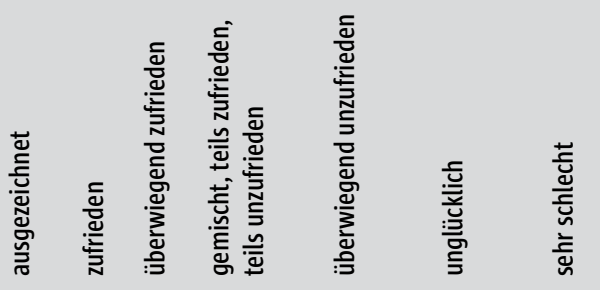

1. Wie würden Sie sich fühlen, wenn sich Ihre jetzigen Symptome beim Wasserlassen in Ihrem weiteren Leben nicht mehr ändern würden?

7. Wie oft sind Sie während des letzten Monats im Durchschnitt nachts aufgestanden, um Wasser zu lassen? Maßgebend ist der Zeitraum vom Zubettgehen bis zum Aufstehen am Morgen.

\begin{tabular}{|c|c|c|c|c|c|c|c|}
\hline $\begin{array}{l}\text { Beeinträchtigung der Lebensqualität } \\
\text { durch Harntraktsymptome }\end{array}$ & 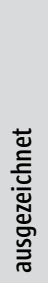 & 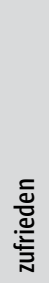 & 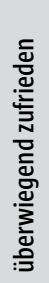 & 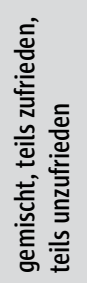 & 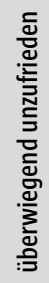 & 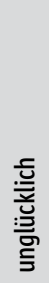 & 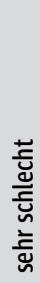 \\
\hline $\begin{array}{l}\text { 1. Wie würden Sie sich fühlen, wenn sich } \\
\text { Ihre jetzigen Symptome beim } \\
\text { Wasserlassen in Ihrem weiteren Leben } \\
\text { nicht mehr ändern würden? }\end{array}$ & 0 & 1 & 2 & 3 & 4 & 5 & 6 \\
\hline
\end{tabular}




\section{Uroflowmetrie}

Mit dieser nicht-invasiven Methode kann die Qualität des individuellen Harnstrahles gemessen und objektiviert werden (s. Abb. 1). Folgende Parameter werden bestimmt:

- Maximaler Harnfluss $\left(\mathrm{Q}_{\max }\right)$

- Durchschnittlicher Harnfluss $\left(\mathrm{Q}_{\text {ave }}\right)$

- Miktionsvolumen (in ml)

- Miktionszeit (in sec)

Das Miktionsvolumen sollte mindestens $150 \mathrm{ml}$ betragen um interpretierbare Parameter zu erhalten. Ein erniedrigter $\mathrm{Q}_{\max }$ ist aber nicht beweisend für eine prostatabedingte Miktionsstörung: dies kann z.B. auch durch eine Meatusstenose, Harnröhrenenge oder Blasenmuskelschwäche bedingt sein. Die Uroflowmetrie dient auch als Instrument der Therapiekontrolle.

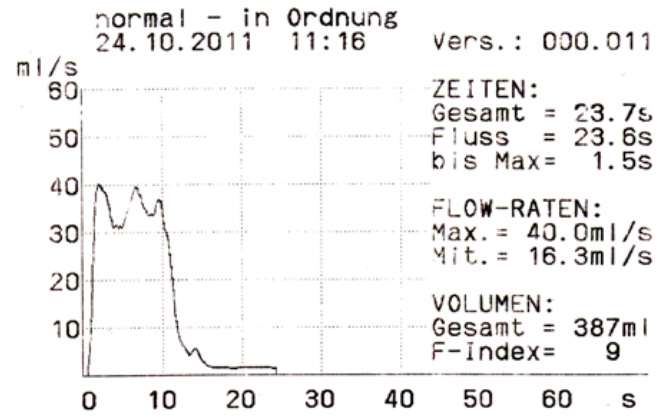

Abb. 1 Normalbefund einer Uroflowmetrie (Bildmaterial: A. Hegele)

\section{Sonografische Untersuchung}

Die sonografische Untersuchung der Nieren und der Prostata im Rahmen der BPSDiagnostik ist obligat. Neben urogenitalen Malignomen sollten eine postrenale Abflussbehinderung und Steine im Urogenitaltrakt ausgeschlossen werden. Die Harnblasenwanddicke kann ebenso wie das Vorhandensein von Blasendivertikeln eruiert werden. Ebenso sollte eine sonografische transabdominelle Bestimmung des Restharns nach erfolgter Spontanmiktion sowohl im Rahmen der primären Diagnostik als auch zur Therapiekontrolle erfolgen. Die Prostata wird mittels des transrektalen Ultraschalls (TRUS) beurteilt. Hier kann im Longitudinal- und Transversalschnitt das Volumen der Prostata sehr exakt bestimmt werden (s. Abb. 2). Neben der Volumenbestimmung können anhand des Echomusters abklärungsbedürftige suspekte Areale lokalisiert werden.

\subsubsection{Sonstige Zusatzdiagnostik}

Sollte aufgrund der durchgeführten Diagnostik nicht geklärt sein, ob eine Miktionsstörung durch eine Obstruktion oder eine eingeschränkte Funktion des M. destrusor vesicae bedingt ist, sollte zur weiteren Klärung eine urodynamische Messung erfolgen 
(z.B. bei Patienten mit einem hohen IPSS aber in der Uroflowmetrie nachgewiesenem kräftigen Harnstrahl $\left(\mathrm{Q}_{\max }\right)$. Eine diagnostische Spiegelung der Harnröhre und der Harnblase sollte immer erfolgen, wenn eine Mikro-/Makrohämaturie bestand und ein Urothelkarzinom der Harnblase ausgeschlossen werden muss. Bei der Untersuchung kann die prostatische Harnröhre mitbeurteilt werden. Eine Standarduntersuchung zur Beurteilung der Therapienotwendigkeit stellt die Urethrozystoskopie nicht dar, kann aber nützliche Informationen liefern (s. Abb. 3). Die radiologische Bildgebung stellt keine Standarddiagnostik dar, der Einsatz muss individuell entschieden werden. Im Rahmen der Abklärung einer Mikro-/Makrohämaturie sollten mittels intravenösem

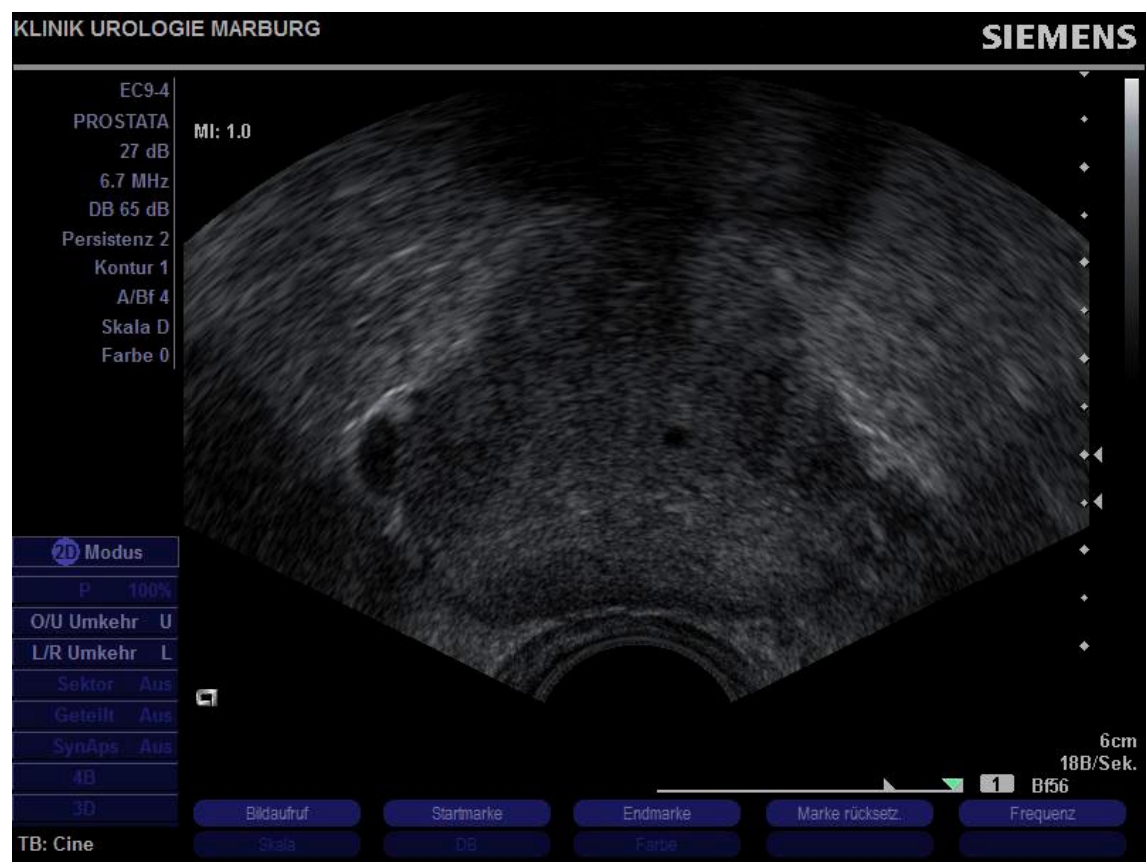

Abb. 2 Darstellung der Prostata mit dem transrektalen Ultraschall (Bildmaterial A. Hegele)
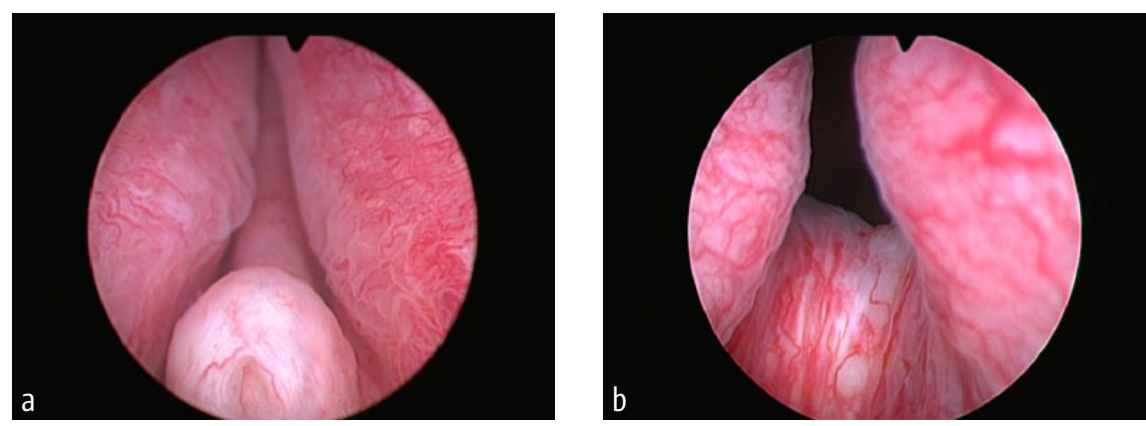

Abb. 3a, b Endoskopisches Bild einer Obstruktion durch deutlich vergrößerte Prostataseitenlappen und eine beginnende Vergrößerung des Prostatamittellappens (Bildmaterial A. Hegele) 
Pyelogramm Tumoren des oberen Harntraktes ausgeschlossen werden, mittels einer retrograden Urethrographie relevante Harnröhrenengen.

\subsubsection{Therapie}

Die BPS kann, nach adäquater abklärender Diagnostik, verschieden therapiert werden. Zur Verfügung stehen, neben der Überwachungsstrategie, verschiedene Medikamente und operative Verfahren. Die Indikation zur jeweiligen Therapie ist maßgeblich von den Symptomen/Komplikationen aber auch von den Wünschen des Patienten, welche sich aus dem Leidensdruck ergeben, abhängig.

\section{Watchful Waiting}

Leidet der Patient unter nicht stark ausgeprägten Symptomen (IPSS < 7) verbunden mit einer vernachlässigbaren Einschränkung der Lebensqualität kann eine Überwachungsstrategie angestrebt werden. Oft können Änderungen des Lebensstils (Trinkmenge, Getränkeart) die Symptome reduzieren. Selbstverständlich ist die regelmäßige Kontrolle und Erweiterung der Therapie bei einer Progression (z.B. Zunahme der Restharnmenge) (Oelke et al. 2010; Wiygul u. Babayan 2009).

\section{Medikamentöse Therapie}

Hier steht eine Vielzahl von Medikamenten zur Verfügung. Diese führen nur bedingt zu einer Desobstruktion, lindern aber meist die Symptome. Zur medikamentösen Therapie sollten nur Substanzen verwendet werden, deren Wirksamkeit in randomisierten und v.a. placebokontrollierten Studien (Placeboeffekt bis 39\%) mit einer ausreichenden Nachbeobachtungszeit erbracht wurde.

\section{Phytotherapeutika}

Phytotherapie ist die arzneiliche Anwendung von Extrakten aus Pflanzen (oder deren Teilen). Hierzu zählen zur Behandlung der BPS z.B. Medikamente aus Kürbiskernextrakten, Brennnesselwurzel, Sägezahnpalmenfrüchten, Roggenpollen, Phytosterolen, Kaktusblütenextrakte und Extrakte aus dem afrikanischen Pflaumenbaum. Obwohl bei diesen Präparaten bisher der Wirksamkeitsnachweis im Rahmen von placebo-kontrollierten Studien nur eingeschränkt erbracht ist, kommen Pflanzenextrakte primär bei LUTS am häufigsten zur Anwendung. Oft handelt es sich um Mischpräparate, sodass die Wirkung nicht eindeutig einer definierten Substanz zugeordnet werden kann. Erfreulicherweise kommt es meist nicht zu relevanten Nebenwirkungen (Oelke et al. 2005, 2010).

\section{a-Blocker}

In der Prostata sind neben Epithel- und Bindegewebszellen auch glatte Muskelzellen vorhanden. Diese finden sich ebenfalls am Blasenhals. Bei einer BPH kommt es zu einer Zunahme der Muskelzellen. Hier finden sich $\alpha$-Adrenorezeptoren, deren Stimulation zu einer Zunahme des urethralen Druckes führen. Die Hemmung dieser Rezeptoren am Blasenhals und im Bereich der Prostata durch $\alpha$-Blocker kann den Druck in der prostatischen Harnröhre und dadurch den Auslasswiderstand senken 
(dynamische Komponente). Die Symptomatik wird durch $\alpha$-Blocker deutlich verbessert, nachgewiesen durch eine Verbesserung des IPSS und des $Q_{\max }$. Vorteile der verschiedenen $\alpha$-Blocker sind die orale Einnahme, teilweise in Retardformulierung und ein rascher Wirkungseintritt innerhalb von wenigen Stunden, welches zu einer hohen Patienten-Compliance führt. Unerwünschte Nebenwirkungen wie Hypotension, orthostatische Dysregulation, Kopfschmerzen, Schwindel treten in bis zu 6o\% der behandelten Patienten auf, abhängig vom verwendeten Präparat und den individuellen Komorbiditäten (Madersbacher et al. 2007; Berges 2006).

\section{5-a-Reduktasehemmer}

Wie bereits erläutert, ist das Wachstum der Prostata androgenabhängig und DHT ist der entscheidende Faktor in der Prostata. Die Umwandlung von Testosteron in DHT kann durch die Hemmung der 5- $\alpha$-Reduktase (Isoenzyme Typ 1 und 2) unterbunden werden. Dies hat zur Folge, dass es innerhalb der Prostata zu einer androgenentzugsbedingten Apoptose kommt, was eine Verkleinerung des Organvolumens (20-30\%) mit konsekutiver Verbesserung der Miktionsbedingungen zur Folge hat (statische Komponente). Der volle Wirkungseintritt erfolgt nach 6 Monaten, die Effekte sind v.a. bei hohem Prostatavolumen (> $40 \mathrm{ml}$ ) ausgeprägt. In Deutschland sind die $5^{-\alpha-}$ Reduktasehemmer Finasterid (hemmt Isoenzym Typ 2) und Dutasterid (hemmt beide Isoenzyme) zur Therapie der BPH zugelassen (orale Medikation). Wichtig ist eine prätherapeutische Bestimmung des PSA-Wertes, da dieser durch die Therapie um bis zu 50\% gesenkt wird. Insgesamt sind beide Medikamente gut verträglich. Nach 1 Jahr Therapie zeigten sich im Vergleich zu einer Placebogruppe eine geringe Zunahme der erektilen Dysfunktion ( $5 \%$ vs. $2 \%$ ), eine Libidoabnahme (4,8\% vs. $2,5 \%$ ) und eine Ejakulationsstörung (3,5\% vs. 1,1\%) (Naslund u. Miner 2007; Marberger 2006).

\section{Kombinationstherapie}

Bei der gleichzeitigen Verwendung von zwei Medikamenten mit unterschiedlicher Wirkweise sollen die jeweiligen Vorteile der einzelnen Therapie kombiniert werden (dynamische und statische Komponente). Hier liegen vielversprechende Daten aus mehreren Studien zur Kombination von $\alpha$-Blockern und 5 - $\alpha$-Reduktasehemmern vor. Es zeigte sich eine Überlegenheit der Kombinationsbehandlung bezüglich $\mathrm{Q}_{\max }$, Risiko des Harnverhaltes und Notwendigkeit einer chirurgischen Intervention (MTOPS-Studie medical therapy of prostatic symptoms + CombAT-Studie; Oelke et al. 2010; McConnell et al. 2003; Roehrborn et al. 2010).

\section{Operative Therapie}

Absolute Operationsindikationen bei einer BPS sind

- wiederkehrende Harnverhalte

- wiederkehrende Harnwegsinfektionen

v nicht beherrschbare Makrohämaturie

- Harnblasensteine

- Dilatation des oberen Harntraktes bis hin zur Niereninsuffizienz

- steigender Restharn unter medikamentöser Therapie 


\section{Transurethrale Resektion der Prostata (TUR-P)}

Die TUR-P stellt den Goldstandard der operativen BPS-Therapie dar. Über eine stromdurchflossene Drahtschlinge wird das Gewebe durchtrennt und es findet eine sofortige Beseitigung der Obstruktion statt. Die gefürchtetste Komplikation ist das sog. TUR-Syndrom in ca. $2 \%$. Hierbei wird Spüllösung über eröffnete Prostatagefäße eingeschwemmt. Folge kann eine hypotone Hyperhydratation mit konsekutiver Bradykardie, Elektrolytverschiebung, Kreislaufdepression sein. Weitere Komplikationen sind die Belastungsinkontinenz ( 2\%), Blasenhalsengen (bis $3 \%$ ), Harnröhrenstrikturen (bis 12\%). Echte Rezidive treten sehr selten auf. Die Re-Operationsrate aufgrund eines Rezidivadenoms beträgt nach 8 Jahren bis zu 15\% (Hegele et al. 2002; Hofmann 2009).

\section{Transvesikale Prostataadenomenukleation (TVP)}

Bei der offenen Adenomenukleation wird das Prostataadenom aus der Kapsel über einen abdominellen Zugang in toto entfernt. Da die TUR-P durch das zu resezierende Gewebe limitiert ist (ca. 80 g) kommt diese Technik vor allem bei größeren Prostatavolumina zum Einsatz.

Beide Operationsverfahren führen zu einer prompten signifikanten Verbesserung des Qmax und des IPSS bei hoher Patientenzufriedenheit (Oelke et al. 2010; Hegele et al. 2002; Hofmann 2009; Roos et al. 1989; Cattolica et al. 1997; WendtNordahl et al. 2009).

\section{Laserverfahren}

\section{Greenlightlaser (Photoselektive Vaporisation der Prostata)}

Die Energie des Laserstrahls (Wellenlänge 532 nm) wird vom Hämoglobin aufgenommen und führt zur Erhitzung des umliegenden Gewebes. Dadurch verdampft das Gewebe. Vorteile sind der Einsatz unter laufender antikoagulatorischer Medikation. Bei dieser Technik kommt es zu keinem TUR-Syndrom; an Komplikationen sind vor allem dysurische Beschwerden und Harnwegsinfekte zu nennen (Heinrich et al. 2007; Schiefelbein 2009).

\section{Holmium:YAG Laser-Enukleation der Prostata (HoLEP)}

Bei einer Wellenlänge von 2.10o nm wird mittels eines Holmium:YAG Lasers das Adenomgewebe, der Prostatakapsel folgend, endoskopisch enukleiert. Das Gewebe wird in der Blase abgeworfen, zerkleinert und entfernt (Kuntz 2009; Gilling et al. 2008).

Die Laserverfahren finden zunehmende Anwendung, die Eruierung von Langzeitdaten im Vergleich zur TUR-P bleibt zukünftigen Untersuchungen vorbehalten (Oelke et al. 2010; Bouchier-Hayes et al. 2006, 2010). 


\section{Literatur}

Alexander RB, Propert KJ, Schaeffer A|, Landis IR, Nickel IC, O'Leary MP, Pontari MA, McNaughton-Collins M, Shoskes DA, Comiter CV, Datta NS, Fowler JE, Nadler RB, Zeitlin SI, Knauss IS, Wang Y, Kusek JW, Nyberg LM, Litwin MS (2004) Ciprofloxacin or Tamsulosin in men with chronic prostatitis/chronic pelvic pain syndrome. Ann Intern Med 141, 581-589

Berges R (2006) Drug therapy for benign prostatic syndrome (BPS). Aktuelle Urol 37, 351-362

Bouchier-Hayes DM, Anderson P, Van Appledorn S, Bugeja P, Costello AJ (2006) KTP laser versus transurethral resection: early results of a randomized trial. I Endourol 20, 580-585

Bouchier-Hayes DM, Van Appledorn S, Bugeja P, Crowe H, Challacombe B, Costello Al (2010) A randomized trial of photoselective vaporization of the prostate using the $80-\mathrm{W}$ potassium-titanyl-phosphate laser vs transurethral prostatectomy, with a 1-year follow-up. BJU Int 105, 964-969

Boyle P (1994) New insights into the epidemiology and natural history of benign prostatic hyperplasia. Prog Clin Biol Res 386, 3-18

Cattolica EV, Sidney S, Sadler MC (1997) The safety of transurethral prostatectomy: a cohort study of mortality in 9416 men. J Urol 158, 102-104

Fitzpatrick JM (2006) The natural history of benign prostatic hyperplasia. BJU Int 97, 3-6

Gilling PI, Aho TF, Frampton CM, King Cl, Fraundorfer MR (2008) Holmium laser enucleation of the prostate: results at 6 years. Eur Urol 53, 744

Hegele A, Zoltan V, Olbert P, Heidenreich A, Hofmann R (2002) Is transurethral resection of large prostatic adenomas justified? Eur Urol 1, 671

Heinrich E, Schiefelbein F, Schön G (2007) Technique and Short-Term Outcome of Green Light Laser Vaporization of the prostate. Eur Urol 52, 1632-1637

Hochreiter WW, Ludwig M, Weidner W, Wagenlehner F, Naber K, Eremenco S, Arnold B (2001) Deutsche Übersetzung des National Institutes of Health Chronic Prostatitis Symptom Index. Urologe A 40, 16-17

Hofmann R (2009) Transurethrale Resektion (TURP) und transurethrale Inzision (TUIP) der Prostata. In: Hofmann R (Hrsg.) Endoskopische Urologie, Springer Heidelberg, 57-96

Kuntz RM (2009) Holmium-Laser-Enukleation der Prostata (HoLEP). In: Hofmann R (Hrsg.) Endoskopische Urologie, Springer Heidelberg, 105-129

Ludwig M, Schroeder-Printzen I, Lüdecke G, Weidner W (2000) Comparison of expressed prostatic secretions with urine after prostatic massage - a means to diagnose chronic prostatitis/inflammatory chronic pelvic pain syndrome. Urology 55, 175-177

Madersbacher S, Marszalek M, Lackner I, Berger P, Schatzl G (2007) The long-term outcome of medical therapy for BPH. Eur Urol 51, 1522-1533

Marberger M (2006) Drug Insight: 5alpha-reductase inhibitors for the treatment of benign prostatic hyperplasia. Nat Clin Pract Urol 3, 495-503

Mayo ME, Ross SO, Krieger JN (1998) Few patients with "chronic prostatitis“ have significant bladder outlet obstruction. Urology 52, 417-421

McConnell ID, Roehrborn CG, Bautista OM, Andriole GL Ir, Dixon CM, Kusek JW, Lepor H, McVary KT, Nyberg LM Ir, Clarke HS, Crawford ED, Diokno A, Foley IP, Foster HE, Jacobs SC, Kaplan SA, Kreder KJ, Lieber MM, Lucia MS, Miller G), Menon M, Milam DF, Ramsdell JW, Schenkman NS, Slawin KM, Smith JA (2003) The long-term effect of doxazosin, finasteride, and combination therapy on the clinical progression of benign prostatic hyperplasia. Medical Therapy of Prostatic Symptoms (MTOPS) Research Group. N Engl I Med 349, 2387-2398

Naslund M), Miner M (2007) A review of the clinical efficacy and safety of 5alpha-reductase inhibitors for the enlarged prostate. Clin Ther 29, 17-25

Nickel JC (1998) Prostatitis: myths and realities. Urology 51, 362-366

Oelke M, Alivizatos G, Emberton M, Gravas S, MAdersbacher S, Michel M, Nordling I, Rioja Sanz C, de la Rosette I (2010) Guidelines on benign prostatic hyperplasia. EAU Guidelines

Oelke M, Berges RR, de la Rosette II (2005) Pharmakologische Therapie des benignen Prostatasyndroms. In: Truß MC, Stief CG, Machtens S, Wagner T, Jonas U (Hrsg.) Pharmakotherapie in der Urologie, Springer Heidelberg, 313-340 
Roehrborn CG, Siami P, Barkin I, Damião R, Major-Walker K, Nandy I, Morrill BB, Gagnier RP, Montorsi F (2010) The effects of combination therapy with dutasteride and tamsulosin on clinical outcomes in men with symptomatic benign prostatic hyperplasia: 4-year results from the CombAT study.; CombAT Study Group. Eur Urol 57, $123-131$

Roos N, Wennber |, Malenka D (1989). Mortality and reoperation after open and transurethral resection of the prostate for benign prostatic hyperplasia. N Engl I Med 320, 1120

Sanda MG, Beaty TH, Stutzman RE, Childs B, Walsh PC (1994) Genetic susceptibility of benign prostatic hyperplasia. J Urol 152, 115-119

Schaeffer A|, Anderson RU, Krieger IN, Lobel B, Naber K, Nakagawa M, Nickel JC, Nyberg L, Weidner W (2006) The Assessment And Management Of Male Pelvic Pain Syndrome Including Prostatitis. In: Mc Connel J, Abrams P, Denis L, Khoury S, Roehrsom C (eds.) Male Lower Urinary Tract Dysfunction. Evaluation and Management 6th International Consultation in Prostate Cancer and Prostate Diseases. Health Publications, Paris, S. 343-385

Schiefelbein F (2009) Greenlight-Laser-Vaporisation der Prostata. In: Hofmann R (Hrsg.) Endoskopische Urologie, Springer Heidelberg, 130-142,

Schneider H, Ludwig M, Hossain HM, Diemer T, Weidner W (2003) The 2001 Giessen Cohort Study on patients with prostatitis syndrome - an evaluation of inflammatory status and search for microorganisms 10 years after a first analysis. Andrologia 35, 258-262

Schneider H, Wilbrandt K, Ludwig M, Beutel M,Weidner W (2005) Prostate-related pain in patients with chronic prostatitis/chronic pelvic pain syndrome. BJU Int 95, 238-243

Shoskes DA, Nickel IC, Dolinga R, Prots D (2009) Clinical Phenotyping of Patients With Chronic Prostatitis/Chronic Pelvic Pain Syndrome and Correlation With Symptom Severity. Urology 73, 538-542

Taylor BC, Noorbaloochi S, McNaughton-Collins M, Saigal CS, Sohn MW, Pontari MA, Litwin MS, Wilt T) (2008) Excessive antibiotic utilization in men with prostatitis. Am I Med 121, 444-449

Wagenlehner FME, Naber KG, Bschleipfer T, Brähler E, Weidner W (2009a) Prostatitis und männliches Beckenschmerzsyndrom. Diagnostik und Therapie. Dtsch Arztebl Int 106, 175-183

Wagenlehner FME, Schneider S, Ludwig M, Schnitker I, Brähler E, Weidner W (2009b) A Pollen Extract (Cernilton) in Patients with Inflammatory Chronic Prostatitis-Chronic Pelvic Pain Syndrome: A Multicentre, Randomised, Prospective, Double-Blind, Placebo-Controlled Phase 3 Study. Eur Urol 56, 544-551

Wendt-Nordahl G, Cao Y, Häcker A, Michel MS, Knoll T (2009) Transurethral resection of the prostate: defending its leading position in the management of benign prostatic enlargement. Minerva Urol Nefrol 61, 291-300

Wiygul J, Babayan RK (2009) Watchful waiting in benign prostatic hyperplasia. Curr Opin Urol 19, 3-6 